XIV Simpósio Nacional de Sistemas Prediais

Gestão, Eficiência e Sustentabilidade

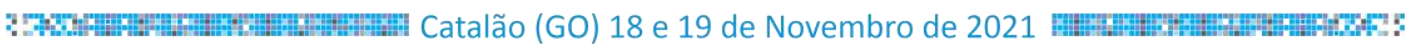

\title{
KITS HIDRÁULICOS PREDIAIS INDUSTRIALIZADOS
}

\author{
Industrialized hydraulic building kits
}

\author{
DI RUZZA, Giulia ${ }^{\text {; }}$ CARDOSO, Luiz Reynaldo²
}

Recebido em 29 de julho de 2021, aprovado em 13 de setembro de 2021, publicado em 18 de novembro de 2021

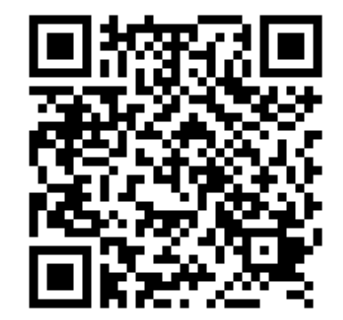

Palavras-chave:

Kits hidráulicos;

Industrialização;

Inovação.

Keywords:

Hydraulic kits;

Industrialization;

Innovation.
RESUMO: A necessidade crescente de industrialização dos processos construtivos faz com que seja necessário modificar a lógica de produção de obras. Isso implica em tornar os processos mais inteligentes e produtivos, reduzir prazos e os desperdícios. Uma maneira de otimizar os processos é por meio da substituição das atividades convencionais por atividades industrializadas que visam a produção acelerada com maior controle de qualidade. Neste contexto, a aplicação de kits hidráulicos produzidos em ambientes fabris controlados se torna um importante objeto de estudo, pois modifica o modelo tradicional produtivo e incrementa tecnologia e inovação. 0 objetivo deste artigo é discutir a implantação de kits hidráulicos prediais industrializados, abordando os tipos existentes no mercado, seus componentes, vantagens, requisitos do sistema e realizando uma análise comparativa sobre a produtividade entre os sistemas industrializados e os convencionais, por meio de um estudo de caso preliminar. A metodologia compõe-se de levantamento bibliográfico e estudo de caso preliminar. Os resultados indicam que a incorporação dessa tecnologia industrializada pode representar um maior grau de eficiência em obras, permitindo maior velocidade de execução, escala produtiva e padronização.

ABSTRACT: The growing need for industrialization of construction processes makes necessary the modification of the logic in production of construction. This implies making processes smarter and more productive, reducing deadlines and wastes. One way to optimize processes is by replacing conventional activities with industrialized ones, aiming for accelerated production with greater quality control. In this context, the application of hydraulic kits produced in controlled manufacturing environments becomes an important subject of study, because it modifies the traditional production model and increments technology and innovation. The goal of this article is to discuss the implementation of industrialized hydraulic building kits, presenting the existing types on the market, the components, benefits, system requirements and performing a comparative analysis about the productivity of the industrialized and conventional systems, through a preliminary study case. The methodology consists of a bibliographic survey and a preliminary case study. The results indicate that the incorporation of this industrialized technology may represent a greater level of efficiency in works, allowing faster execution speed, productive scale and standardization.

\section{CONTATO DOS AUTORES:}

${ }^{1}$ DI RUZZA, Giulia: Escola Politécnica da Universidade de São Paulo (USP), giuliadiruzza@usp.br

${ }^{2}$ CARDOSO, Luiz Reynaldo: Escola Politécnica da Universidade de São Paulo (USP), luizcardoso@usp.br 


\section{INTRODUÇÃO}

A industrialização dos processos construtivos e a aplicação de modos de produção acelerada por meio da mecanização intensiva de tarefas faz com que seja possível construir mais em menos tempo. Nesse sentido, é necessário modificar a lógica de produção atual de obras, o que requer maior grau de planejamento e logística dentro do canteiro de obras, sendo possível agregar mais agilidade e controle de qualidade. Tudo isso reflete a principal tendência do mercado contemporâneo: a indústria 4.0.

Entretanto, as práticas construtivas atuais ainda não refletem os avanços tecnológicos experimentados pelos demais setores. A produção tradicional é ainda muito atrelada à utilização de técnicas com composição artesanal, que dependem da habilidade da mão de obra, onde há maior dificuldade de controle, organização, maior número de operações nos canteiros de obras e maior número de pessoas trabalhando em campo.

De acordo com Barros (2013) "produzir em grande escala, com baixo custo e em curto prazo são os desafios frente aos quais as organizações identificaram claramente que não seria possível vencê-los utilizando-se da mesma base tecnológica das últimas décadas [...]". Sendo assim, a incorporação de tecnologias e a adoção de modos de produção industrializada e racionalizada encontram oportunidades de crescimento dentro do mercado como formas de atingir as novas necessidades de otimização da construção civil.

Neste contexto, os sistemas prediais hidráulicos demonstram ser um subsistema dos edifícios que precisa ser aprimorado, visto a quantidade elevada de patologias que costumam ser apontadas após a entrega dos empreendimentos. Assim, os kits hidráulicos prediais industrializados se apresentam como uma alternativa para atingir um controle de qualidade mais apurado, com desempenho assegurado pelos testes realizados dentro da indústria, maior padronização, produtividade, entre diversos outros fatores a serem discutidos.

O principal objetivo deste trabalho é discutir a implantação dos kits hidráulicos prediais industrializados como inovações tecnológicas na construção civil. O objetivo secundário é sistematizar os conhecimentos sobre a utilização dos kits hidráulicos como uma alternativa construtiva industrializada, capaz de agregar valor e inovação no setor da construção civil.

\section{MATERIAL E MÉTODOS}

O trabalho apresenta inicialmente um levantamento bibliográfico para detalhar informações atuais sobre a construção hidráulica convencional e a construção com os kits hidráulicos prediais industrializados, abordando os componentes dos sistemas, processo de montagem até instalação nos canteiros de obra, vantagens e requisitos do sistema.

Em seguida, o trabalho apresenta um estudo de caso preliminar de um empreendimento residencial de alto padrão situado na cidade de São Paulo, onde será avaliada a produtividade do sistema convencional e do sistema industrializado, composto pelos kits.

O estudo de caso preliminar foi desenvolvido em um apartamento modelo de $160 \mathrm{~m}^{2}$, onde foram levantados os dados referentes às instalações hidráulicas convencionais e industrializadas de apenas cinco ambientes, sendo eles: quatro banheiros e uma área de serviço. Os dois tipos de instalações foram executados em um mesmo modelo de apartamento, pela mesma equipe, sendo que todos os funcionários haviam recebido os devidos treinamentos antes do início dos serviços. 
Para o levantamento da produtividade da construção com os kits, os mesmos foram instalados em cinco ambientes do apartamento modelo, sendo: quatro kits banheiros (um em cada banheiro da unidade) e um kit área de serviço. Já para o levantamento da produtividade da construção sem os kits, a construção convencional foi adotada para as instalações hidráulicas dos cinco ambientes mencionados.

O levantamento foi realizado através da medição do tempo que a equipe precisou para executar a instalação dos kits e as instalações hidráulicas convencionais nos ambientes determinados, a fim de avaliar a produtividade das duas metodologias construtivas.

\section{REVISÃO BIBLIOGRÁFICA}

\subsection{Contextualização}

Os sistemas prediais hidráulicos apresentam um alto índice de assistência técnica no pós venda, sendo o subsistema da construção que mais apresenta patologias após a entrega dos empreendimentos. É possível destacar algumas causas para o surgimento das patologias nos sistemas hidráulicos convencionais: erros de projeto ou de execução, falhas nos componentes, manutenção inexistente ou irregular e processo de desgaste dos materiais. No sistema convencional, os componentes dos sistemas hidráulicos são adquiridos separadamente e montados ou construídos no local da obra, o que requer um gerenciamento muito maior, por parte da construtora, nos quesitos materiais e mão de obra.

Sendo assim, a produção convencional se torna muito atrelada à utilização de técnicas com composição artesanal, que dependem da habilidade da mão de obra disponível nos canteiros, estando sujeita a erros, maiores desperdícios materiais, padronização e controle de qualidade não muito apurados.

De acordo com Oliveira (2002) "industrializar-se, para a construção, é sinônimo de evoluir". Sendo assim, a industrialização é um processo evolutivo que por meio da implementação de novas tecnologias objetiva incrementar a produtividade. Oliveira (2002) ainda discute a industrialização como um conceito que pressupõe organização, planejamento, continuidade executiva, repetição e eficiência no processo de produção, sendo que sua principal ferramenta é a racionalização.

Segundo Sabbatini (1989), a racionalização construtiva é um processo composto pelo conjunto das ações que tenham por objetivo otimizar o uso de recursos materiais, humanos, organizacionais, energéticos, tecnológicos, temporais e financeiros disponíveis na construção em todas as suas fases.

De acordo com Barros (2013) "não há dúvidas de que o modelo industrial de produção seriada pode e deve inspirar a indústria da construção. No entanto, esse modelo é muito mais do que a simples adoção do que se pode imaginar uma nova tecnologia". Assim sendo, a adoção de tecnologias industrializadas e racionalizadas envolve também uma mudança de mentalidade, sendo necessário modificar a lógica de produção de obras.

De acordo com Aro (2004), a partir de 1990, o setor da construção civil brasileiro passou a contar com novidades provindas da indústria da pré-fabricação importadas da Europa e da América, surgindo como alternativa construtiva os kits hidráulicos para os sistemas prediais hidráulicos e sanitários. 
Nesse sentido, a produção off-site (executada fora do canteiro, dentro das fábricas) garante maior segurança e qualidade dos sistemas, o que é possível verificar com a utilização dos kits hidráulicos industrializados, que surgiram para suprir essa necessidade de melhorar a qualidade das instalações, acelerar e padronizar o processo de montagem.

\subsection{Kits hidráulicos prediais industrializados}

Os conjuntos dos kits hidráulicos prediais industrializados são compostos por tubos, conexões, válvulas e acessórios que reproduzem as instalações hidráulicas completas, além da estrutura de fixação (alguns modelos contam com estruturas metálicas ou plásticas para fixação em alvenaria ou em drywall). De acordo com Aro (2004), os kits hidráulicos industrializados são caracterizados por conjuntos de tubos com cortes prévios e montagem fora do local de alocação das peças, facilitando a sua instalação.

Existem diversos tipos de kits hidráulicos: kit lavatório, kit área de serviço, kit banheiro, kit chuveiro, kit chicote, kit esgoto, kit prumadas, kit abrigo para medidores, entre diversos outros disponíveis no mercado.

Os kits são usados, principalmente, quando a repetição do sistema é elevada, como em edifícios e conjuntos habitacionais. Eles devem ser previstos na etapa de projeto, com a definição dos requisitos técnicos, customização conforme cada empreendimento e é indicado que seja realizada a elaboração prévia de um protótipo do kit para testes e validação da construtora e incorporadora.

Os kits não são normalizados, ou seja, não existem normas técnicas específicas para eles, mas seus componentes e suas instalações devem respeitar as normas vigentes da ABNT, garantindo o desempenho estabelecido pela NBR 15575-6 (ABNT, 2013), NBR 5626 (ABNT, 2020) e a NBR 8160 (ABNT, 1999).

\subsection{Fabricação na indústria a montagem no canteiro: vantagens e requisitos do sistema}

Os kits hidráulicos prediais industrializados são produzidos em fábricas, com produção em alta escala e testados de forma rigorosa. A grande maioria dos fabricantes do setor anuncia que realizam teste de estanqueidade em $100 \%$ dos conjuntos e não apenas em um percentual de amostragem.

Após serem testados, são protegidos e embalados de forma adequada, recebendo lacre de garantia e etiqueta de rastreamento. Posteriormente, são transportados para os canteiros de obra conforme o cronograma e programação de entrega, onde a instalação é realizada por uma equipe de obra treinada ou pela própria fornecedora e instaladora.

O material deve ser entregue de forma organizada e identificada nos canteiros, com a sinalização do modelo, local e ambiente de instalação. $O$ armazenamento também exige cuidados, devendo-se respeitar o número máximo de empilhamento dos kits. A sua instalação não é uma etapa complexa, sendo necessário que as equipes responsáveis recebam um treinamento básico e orientações a respeito do uso e manuseio do produto. Os kits são totalmente produzidos em fábrica, exigindo apenas que sejam conectados nas prumadas, interligando os andares superiores aos inferiores.

De acordo com Colinart, Bendouma e Glouannec (2019), o uso de elementos pré-fabricados e oriundos da indústria são capazes de apresentar inúmeras vantagens, podendo-se citar a racionalização e industrialização dos processos de fabricação, maior padronização dos 
sistemas, melhor controle de qualidade e desempenho assegurado por testes, com consequente melhoria na qualidade do produto.

Além disso, é possível mencionar a eliminação de algumas etapas de trabalho dentro dos canteiros, reduzindo tempo, garantindo maior velocidade de instalação, redução do desperdício de peças, menor geração de resíduos, maior sustentabilidade, garantia do sistema assegurada pelo fabricante no pós-venda, possibilidade de rastreamento dos lotes, sendo possível identificar em uma base de dados os locais onde cada kit produzido foi instalado e apresentando expressivo aumento da produtividade.

De acordo com Martins, Hernandez e Amorim (2003), a utilização dos kits hidráulicos em obras é considerada uma boa prática de gestão para prevenir patologias do sistema, além de poder acarretar em maiores ganhos de produtividade e qualidade se comparado ao sistema convencional construtivo.

Monteiro (2017) realizou um estudo exploratório para mensurar a produtividade dos kits hidráulicos, no qual foi destacado que em situações ideais, os kits atingiram produtividade 3,5 vezes maior que produtividade da mão de obra do sistema convencional, demonstrando um ganho de eficiência significativo.

O sistema industrializado também exige requisitos, como: necessidade de especificação adequada e detalhamentos antecipados na etapa de projeto, controle, planejamento e definição de cronograma de obra mais detalhado para a encomenda e recebimento dos materiais. Segundo Barros e Cardoso (2011), é preciso repensar o planejamento e logística dos canteiros, definindo previamente os processos de construção.

\section{RESULTADOS E DISCUSSÃO}

\subsection{Estudo de caso: produtividade dos kits}

Nesta seção, este trabalho apresenta a discussão e os resultados obtidos com o estudo de caso preliminar, que será desenvolvido de forma mais detalhada e aprofundada em pesquisas futuras.

Trata-se de um estudo desenvolvido em uma obra de alto padrão na cidade de São Paulo, de uma empresa Incorporadora. O perfil do empreendimento apresenta duas torres, com quatro apartamentos em cada pavimento e 30 pavimentos residenciais. Para este empreendimento, foram contratados dois tipos de kits hidráulicos prediais industrializados: kit área de serviço e kit banheiro.

Conforme os detalhamentos da seção 2. MATERIAL E MÉTODOS, foi realizado o levantamento em campo e os resultados obtidos são descritos a seguir.

- No sistema convencional, a montagem das instalações hidráulicas dos cinco ambientes especificados do apartamento modelo é realizada em três dias de trabalho. Cada dia de trabalho com 8 horas trabalhadas, totaliza $\mathbf{2 4}$ horas para a montagem das instalações hidráulicas.

- Com os kits hidráulicos industrializados, a montagem das instalações hidráulicas dos mesmos cinco ambientes é realizada em apenas 6 horas de trabalho, ou seja, cada kit é instalado em, aproximadamente, $1 \mathrm{~h}$ e $12 \mathrm{~min}$. 
Desta forma, de acordo com os levantamentos coletados neste estudo de caso preliminar, é possível dizer que a instalação dos kits hidráulicos industrializados foi cerca de 4 vezes mais rápida, representando uma economia de $75 \%$ do tempo em relação ao sistema convencional construtivo. Vale ressaltar que este estudo não possuí tratamento estatístico, sendo que serão realizados levantamentos adicionais em pesquisas futuras, a fim de calibrar os resultados apresentados inicialmente.

\section{CONSIDERAÇÕES FINAIS}

O uso dos kits hidráulicos prediais industrializados representa um avanço para o setor em direção à industrialização da construção, sendo uma alternativa promissora para substituição dos sistemas convencionais.

A implantação dos kits em obras requer um detalhamento de todas as etapas do processo. A produção desses componentes em ambientes controlados pode representar um aumento da produtividade, redução do desperdício e geração de entulho, possibilidade de rastreamento dos lotes e otimização do fluxo dos serviços dentro do canteiro.

Ressalta-se que é importante modificar a lógica de produção de obras, tornando-as um local de montagem e não de produção, transformando uma realidade que ainda é marcada pelo desperdício de materiais e de componentes, dificuldade de controle e organização, retrabalho, e número elevado de operações dentro do canteiro, repensando futuros projetos, investindo em industrialização e inovação.

Assim sendo, a incorporação dessa tecnologia pode anunciar um maior grau de eficiência em obras executadas em todo o país, permitindo maior velocidade de execução, escala produtiva, padronização apurada e agregando competitividade no setor da Construção Civil.

Através dos resultados apresentados no estudo de caso proposto, pode-se afirmar que a construção industrializada com os kits hidráulicos foi cerca de 4 vezes mais rápida do que a construção convencional. Este resultado se aproxima do estudo exploratório realizado por Monteiro (2017), no qual chegou-se em um índice de produtividade de 3,5 vezes maior com a utilização dos kits. Ambos os resultados obtidos demonstram um ganho expressivo de eficiência, portanto, sugere-se o prosseguimento de estudos sobre o tema, em busca de um entendimento mais apurado e resultados precisos através de um tratamento estatístico ampliado.

\section{REFERÊNCIAS}

ARO, Celso Ricardo. A modernização tecnológica: seu patamar nos sistemas prediais hidráulicos e sanitários. Dissertação (mestrado) - Universidade Federal de São Carlos, 2004.

ASSOCIAÇÃO BRASILEIRA DE NORMAS TÉCNICAS. NBR5626: Sistemas prediais de água fria e água quente - Projeto, execução, operação e manutenção. Rio de Janeiro, 2020.

ASSOCIAÇÃO BRASILEIRA DE NORMAS TÉCNICAS. NBR8160: Sistemas Prediais de Esgoto Sanitário Projeto e execução. Rio de Janeiro, 1999.

ASSOCIAÇÃO BRASILEIRA DE NORMAS TÉCNICAS. NBR15575: Edificações habitacionais Desempenho. Rio de Janeiro, 2013. 
BARROS, Mercia M. S. B. Tecnologias construtivas para produção de edifícios no Brasil: perspectivas e desafios. Departamento de Engenharia de Construção Civil. Escola Politécnica USP, 2013.

BARROS, M. M. S. B.; CARDOSO, F. F. Inovação: espiral ou carrossel do conhecimento?. Conjuntura da Construção, São Paulo, p. 10 - 11, 01 jun. 2011.

COLINART, Thibaut; BENDOUMA, Mathieu; GLOUANNEC, Patrick. Building renovation with prefabricated ventilated façade element: A case study. Elsevier - Energy \& Buildings, 2019.

MARTINS, M. S; HERNADES, A. T.; AMORIM, S. V. Ferramentas para melhoria do processo de execução dos sistemas hidráulicos prediais. In: III SIBRAGEC - Simpósio Brasileiro de Gestão e Economia da Construção, 16-19 set. 2003, São Carlos. Anais, sn.

MONTEIRO, Paulo Roberto Moreira. Produtividade da mão de obra na execução de instalações prediais hidráulicas e sanitárias. 2017. Dissertação (Mestrado) - Universidade Federal de São Carlos, 2017.

OLIVEIRA, Luciana Alves de. Tecnologia de painéis pré-fabricados arquitetônicos de concreto para emprego em fachadas de edifícios. 2002. Dissertação (Mestrado) - Escola Politécnica da Universidade de São Paulo, 2002.

SABBATINI, Fernando Henrique. Desenvolvimento de métodos, processos e sistemas construtivos: formulação e aplicação de uma metodologia. 1989. Tese (Doutorado em Engenharia de Construção Civil e Urbana) - Escola Politécnica, Universidade de São Paulo, São Paulo, 1989. 